\title{
A Quantum Field Theory Description of Separate Electric and Magnetic Effects in Elementary Fermion Epigenetics
}

\author{
Claudio Verzegnassi ${ }^{1,2}$ \\ ${ }^{1}$ Department of Chemistry, Physics and Environment, University of Udine, Italy \\ ${ }^{2} \mathrm{AMeC}$ (Association for Medicine and Complexity), Trieste, Italy \\ Email: claudio@ts.infn.it
}

Received 4 January 2015; accepted 24 January 2015; published 28 January 2015

Copyright (C) 2015 by author and Scientific Research Publishing Inc.

This work is licensed under the Creative Commons Attribution International License (CC BY). http://creativecommons.org/licenses/by/4.0/

(c) (i) Open Access

\begin{abstract}
The separate effects that an electric and a magnetic field would have on the total energy and spin of an elementary electron state have been computed in a theoretical quantum field theory framework. It is shown that all the effects in this process, that are defined "fermion epigenetics", can be expressed in a simple and elegant way in terms of the components of the electron field, called "psinons" in this approach. In the minimal interaction prescription, the electric and the magnetic effects can be separated into the sum of "classical" components reproducing conventional Stark and Zeeman effects, and new components of different type. In the non-relativistic limit, the two residual effects on the energy only depend on the electron intrinsic properties, i.e. its charge and its spin, and on the value of the electric and magnetic potentials. A comparison with the results obtainable in a Pauli formalism approach is discussed and, finally, a very qualitative calculation of the size of possible effects is performed.
\end{abstract}

\section{Keywords}

\section{Elementary Fermion Epigenetics}

\section{Introduction}

In two previous papers [1] [2] a definition and a description of "fermion epigenetics" have been proposed in the framework of quantum field theory, concentrating the calculation on the case of one electron state. The motivations of my definition have been thoroughly elucidated in ref. [1] [2], to which I refer for a more complete discussion. For the purpose of this paper it will be enough to recall the fact that the starting point was an 
impressive analogy between the four components of the elementary organic nucleus, the hystones, and the four components of the fermionic field $\psi=\left(\psi_{1}, \psi_{2}, \psi_{3}, \psi_{4}\right)$ that were called "psinons". As shown in [1] [2], all the physical properties of the free electron can be expressed in terms of different combinations of couples of psinons and of their complex conjugate entities.

In this approach, the process of "magnetic fermion epigenetics" has been described as the result of an interaction between the electron and a classical magnetic field treated in the conventional "minimal interaction" scheme. The main motivation for this search was the idea of proposing possible analogies between the results that we obtained and those of a performable future realistic medical study of magnetic effects on the human organism, that have attracted great interest in recent studies. All the obtained results have been concentrated on the determination of "pure magnetic" effects both in the absence [1] and in the presence [2] of surrounding environment. To simplify the calculations, the analysis was limited to the case of a constant magnetic field whose direction was chosen along the $x^{3}$ axis. The results that we have obtained, and the associated discussion on the possibility of analogy with a proper medical experiment, have been discussed in [1] [2].

The aim of this paper is to generalize the investigation of [1] [2] to the case of a non-constant magnetic and a non-constant electric field, whose effects on the total electron energy and spin will be computed to lowest order in the fields, in the minimal prescription approach. The results will first be given in the general case and then in the constant field case. For the sake of completeness, the various expressions will be compared with those that would have been obtained in the conventional Pauli approach, showing analogies and differences. To conclude, a very qualitative numerical estimate of the electric and magnetic effects for constant fields will be performed. A final discussion on the possible relevance of my calculations will then be given at the end of the paper.

\section{Electric and Magnetic Effects on an Elementary Electron State}

I shall start this analysis writing the explicit expressions of the total energy, of the spin and of the electric charge of a free electron state. The reason of the choice of the two "intrinsic" electron properties, spin and charge, will become clear in the following part of the paper. I shall choose as basic quantities, as done in [1] [2], the four complex components of the electron field $\psi(t, \boldsymbol{x})$, i.e. $\psi_{s}(t, \boldsymbol{x}), s=1,2,3,4$, and retain for them my definition of "psinons". For the total energy $\mathcal{H}$ I write:

$$
\begin{aligned}
\mathcal{H}= & \int \mathrm{d}^{3} x\left\{2 m \operatorname{Re}\left(\psi_{1}^{*} \psi_{3}+\psi_{2}^{*} \psi_{4}\right)-2 \operatorname{Im}\left[\psi_{1}^{*} \partial_{3} \psi_{1}+\psi_{1}^{*}\left(\partial_{1}-i \partial_{2}\right) \psi_{2}\right.\right. \\
& \left.+\psi_{2}^{*}\left(\partial_{1}+i \partial_{2}\right) \psi_{1}-\psi_{2}^{*} \partial_{3} \psi_{2}\right]+2 \operatorname{Im}\left[\psi_{3}^{*} \partial_{3} \psi_{3}+\psi_{3}^{*}\left(\partial_{1}-i \partial_{2}\right) \psi_{4}\right. \\
& \left.\left.+\psi_{4}^{*}\left(\partial_{1}+i \partial_{2}\right) \psi_{3}-\psi_{4}^{*} \partial_{3} \psi_{4}\right]\right\}
\end{aligned}
$$

For the spin vector $\boldsymbol{S}$ I have:

$$
S=\int \mathrm{d}^{3} x \boldsymbol{s}
$$

where the "spin current" components $s_{1,2,3}$ are

$$
\begin{aligned}
& s_{1}=\operatorname{Re}\left(\psi_{1}^{*} \psi_{2}+\psi_{3}^{*} \psi_{1}\right), \\
& s_{2}=\operatorname{Im}\left(\psi_{1}^{*} \psi_{2}+\psi_{3}^{*} \psi_{1}\right), \\
& s_{3}=\frac{1}{2}\left(\psi_{1}^{*} \psi_{1}-\psi_{2}^{*} \psi_{2}+\psi_{3}^{*} \psi_{3}-\psi_{4}^{*} \psi_{4}\right)
\end{aligned}
$$

The last expression that is needed is that of the electric charge $Q$. From the conventional definition one obtains

where

$$
Q=e \int \mathrm{d}^{3} x \rho_{Q}(t, x)
$$

$$
\rho_{Q}(t, \boldsymbol{x})=\psi_{1}^{*} \psi_{1}+\psi_{2}^{*} \psi_{2}+\psi_{3}^{*} \psi_{3}+\psi_{4}^{*} \psi_{4}
$$

and $e$ is the electric charge, fixed by the chosen convention, e.g. negative for electrons. The effects on the energy of a classic electromagnetic field, described in the usual way by four potentials $A_{0}, A_{1}, A_{2}, A_{3}$ have been 
now considered. The prescription has been that of the "minimal interaction" approach in which the replacement is used

$$
\partial_{\mu} \Rightarrow \partial_{\mu}-i e A_{\mu}
$$

Imposing the electron field to satisfy the Dirac equation leads then to the following set of changes of the four psinons, denoted as $\Delta_{A} \psi_{s}$ as in [2], that I write for completeness

$$
\begin{aligned}
& \Delta_{A} \psi_{1}=\frac{|e|}{m}\left(A_{0} \psi_{3}+A_{1} \psi_{4}-i A_{2} \psi_{4}+A_{3} \psi_{3}\right), \\
& \Delta_{A} \psi_{2}=\frac{|e|}{m}\left[A_{0} \psi_{4}+A_{1} \psi_{3}+i A_{2} \psi_{3}-A_{3} \psi_{4}\right], \\
& \Delta_{A} \psi_{3}=\frac{|e|}{m}\left[A_{0} \psi_{1}-A_{1} \psi_{2}+i A_{2} \psi_{2}-A_{3} \psi_{1}\right], \\
& \Delta_{A} \psi_{4}=\frac{|e|}{m}\left[A_{0} \psi_{2}-A_{1} \psi_{1}-i A_{2} \psi_{1}+A_{3} \psi_{2}\right] .
\end{aligned}
$$

where, $m$ is the electron mass. The effect of $\Delta_{A} \mathcal{H}$ of the electric and magnetic fields on the energy can now be simply estimated introducing the modified psinons $\psi=\psi_{0}+\Delta \psi, \psi_{0}$ being the free psinons. After a straightforward calculation one finds two separate effects, an electric one and a magnetic one. The electric effect $\Delta_{A_{0}} \mathcal{H}$, contains the electric potential $A_{0}$ and the electric field $\boldsymbol{E}=-\nabla A_{0}$. The magnetic one $\Delta_{A} \mathcal{H}$ depends on the magnetic potential $\boldsymbol{A}$ and on the magnetic field $\boldsymbol{H}=\nabla \wedge \boldsymbol{A}$. Let us begin with the electric effect

$$
\Delta_{A_{0}} \mathcal{H}=|e| \int \mathrm{d}^{3} x\left[A_{0} \rho_{Q}-\boldsymbol{E} \cdot \rho_{\mathrm{E}},\right]
$$

where $\rho_{E}$ is a three-component quantity, whose explicit expression in terms of the four psinons is:

$$
\rho_{E}=\frac{i}{m} \psi^{\dagger} \gamma \psi=\frac{2}{m}\left(\operatorname{Im}\left(\psi_{4}^{*} \psi_{1}+\psi_{3}^{*} \psi_{2}\right), \operatorname{Re}\left(\psi_{4}^{*} \psi_{1}-\psi_{3}^{*} \psi_{2}\right),-\operatorname{Im}\left(\psi_{1}^{*} \psi_{3}+\psi_{4}^{*} \psi_{2}\right)\right]
$$

where $\gamma$ are the conventional gamma matrices. As one notices, the second term in the electric component Equation (13) has an expression that recalls the classic conventional expression of an electric Stark effect. The first term has also a classic analogy with a potential-charge energy effect. The purely magnetic effect can also be computed with a longer but still straightforward work, which produces two different contributions that have been written separately in what follows

$$
\Delta_{\boldsymbol{A}} \mathcal{H}=\left[\Delta_{\boldsymbol{A}} \mathcal{H}\right]_{(1)}+\left[\Delta_{\boldsymbol{A}} \mathcal{H}\right]_{(2)}
$$

Here

$$
\left[\Delta_{\boldsymbol{A}} \mathcal{H}\right]_{(1)}=2|e| \int \mathrm{d}^{3} x \boldsymbol{A} \cdot \boldsymbol{J}
$$

where

$$
\boldsymbol{J}=\bar{\psi} \gamma \psi=\left[\operatorname{Re}\left(-\psi_{1}^{*} \psi_{2}+\psi_{3}^{*} \psi_{1}\right), \operatorname{Im}\left(-\psi_{1}^{*} \psi_{2}+\psi_{3}^{*} \psi_{1}\right), \operatorname{Re}\left(-\psi_{1}^{*} \psi_{1}+\psi_{2}^{*} \psi_{2}+\psi_{3}^{*} \psi_{3}-\psi_{4}^{*} \psi_{4}\right)\right]
$$

The next term can be written as

where

$$
\left[\Delta_{A} \mathcal{H}\right]_{(2)}=-2 \frac{|e|}{m} \int \mathrm{d}^{3} x \boldsymbol{H} \cdot \boldsymbol{\rho}_{H}
$$

$$
\rho_{H}=-\psi^{\dagger} \gamma^{5} \gamma \psi=2\left[\operatorname{Re}\left(\psi_{1}^{*} \psi_{4}+\psi_{2}^{*} \psi_{3}\right), \operatorname{Im}\left(\psi_{1}^{*} \psi_{4}-\psi_{2}^{*} \psi_{3}\right), \operatorname{Re}\left(-\psi_{1}^{*} \psi_{3}-\psi_{2}^{*} \psi_{4}\right)\right]
$$

Equations (13), (16), (18) provide the electric and magnetic effects on the electron energy. The effects have been computed to lowest order in the in the electromagnetic potentials, neglecting components $\sim A_{\mu} A_{\nu}$. The psinons that appear in all the equations are free psinons. One sees thus that all the computed effects depend on the product of the electric and magnetic potentials with some properties of the free electron system, specified by 
different psinon couples combinations. In the magnetic effect one can still recognize a classic term of "Zeeman effect" kind, precisely the term, associated to a "magnetic dipole moment" $\simeq \rho_{H}$. The remaining term is of different kind, and contains a scalar product of the magnetic potential $\boldsymbol{A}$ with free electron vector quantities. I have then examined what remains of the effects in the non-relativistic limit, that seems to be more relevant for medical weak-field searches. In this limit (NRL) the two effects are greatly reduced, accepting the (Dirac) condition

$$
\psi_{3,4} \ll \psi_{1,2}
$$

From this condition one easily finds that the two surviving terms have the following form in the limit

$$
\begin{aligned}
& \Delta_{A_{0}} \mathcal{H}_{N R L} \rightarrow|e| \int \mathrm{d}^{3} x A_{0} \rho_{Q(N R L)} \\
& \Delta_{\boldsymbol{A}} \mathcal{H}_{(N R L)} \rightarrow-4|e| \int \mathrm{d}^{3} x \boldsymbol{A} \cdot \boldsymbol{s}_{(N R L)}
\end{aligned}
$$

In Equations (21), (22) the expressions of the charge density $\rho_{Q(N R L)}$ and of the spin density $s$ are those which survive in the non-relativistic limit, i.e.

$$
\begin{gathered}
\rho_{Q(N R L)}=\psi_{1}^{*} \psi_{1}+\psi_{2}^{*} \psi_{2} \\
\boldsymbol{s}_{(N R L)}=\left[\operatorname{Re}\left(\psi_{1}^{*} \psi_{2}\right), \operatorname{Im}\left(\psi_{1}^{*} \psi_{2}\right), \frac{1}{2}\left(\psi_{1}^{*} \psi_{1}-\psi_{2}^{*} \psi_{2}\right)\right]
\end{gathered}
$$

Equations (21), (22) represent the main results that have been obtained. They are valid for general, i.e. nonconstant fields. This case can be easily treated replacing in the first term of the magnetic effect equation (22) the magnetic potential potential $\boldsymbol{A}$ with

$$
\boldsymbol{A} \Rightarrow \frac{1}{2} \boldsymbol{H} \wedge \boldsymbol{x}
$$

One obtains in this case an overall contribution of the kind $|e| \int \mathrm{d}^{3} \boldsymbol{x} \boldsymbol{H} \cdot \boldsymbol{M}_{H}$ where the "magnetic dipole" term $\boldsymbol{M}_{H}$ has a certain expression that also depends explicitly on the integration variable $\boldsymbol{x}$.

Returning to the NRL, one sees that the two effects are given by the product of the potentials $A_{0}, \boldsymbol{A}$ with the two intrinsic properties of the free electron, i.e. its charge and its spin. Clearly, for given electromagnetic potentials, the intrinsic properties of the electron determine the effects. Alternatively, for identical electron intrinsic properties, a variation of the input will change drastically the result. As a simple and possibly relevant example, a rotation of the magnetic potentials may change the effect in a visible (and verifiable) way. In particular, inverting the direction of the magnetic potential, and therefore that of the magnetic field which is orthogonal to $\boldsymbol{A}$, would change the sign of the effect. This might lead to the possibility of producing and "optimal" effect, which might be the goal of future dedicated medical experiments.

A final point to be discussed is the fact that the formal expressions of the general electric and magnetic effects, computed adopting the minimal interaction formalism and exploiting the Dirac equation, appear perhaps different from what one would expect on the basis of the analogy with the simple Stark and Zeeman effects. In this spirit I have computed the effects that would have been produced in the quantum field theory framework replacing the minimal interaction approach with a Pauli scheme, in which one simply adds to the Lagrangian the interaction term

$$
L_{\text {Pauli }}=\mu \bar{\psi} \sigma^{\mu v} \psi F_{\mu v}
$$

where $F_{\mu v}=\partial_{\mu} A_{\nu}-\partial_{v} A_{\mu}$ and $\sigma^{\mu v}=\frac{i}{2}\left[\gamma^{\mu}, \gamma^{v}\right]$. The calculation of the energy shift are much simpler in this case. Denoting the effect on the Hamiltonian as $\Delta_{\mu} \mathcal{H}$, one finds that:

$$
\Delta_{\mu} \mathcal{H}=\mu \int \mathrm{d}^{3} x\left[\boldsymbol{E} \cdot \boldsymbol{J}_{E}+\boldsymbol{H} \cdot \boldsymbol{J}_{H}\right]
$$

Equation (27) reproduces the effects of conventional Stark and Zeeman type, with the explicit expression for the two currents, that I write for simplicity in a conventional way as

$$
\boldsymbol{J}_{E}=i \bar{\psi}\left(\begin{array}{cc}
\sigma & 0 \\
0 & -\sigma
\end{array}\right) \psi
$$




$$
\boldsymbol{J}_{H}=\bar{\psi} \boldsymbol{\Sigma} \psi=i \bar{\psi}\left(\begin{array}{cc}
\sigma & 0 \\
0 & \boldsymbol{\sigma}
\end{array}\right) \psi
$$

where the $\sigma^{i}$ are the Pauli matrices. In other words, one of the components in the minimal interaction scheme coincides with the Pauli term, with the request that

$$
\mu=-\frac{e}{m}=-2\left[\frac{e}{2 m}\right]
$$

A possible conclusion is that the minimal interaction scheme produces in the general case the effects that one would expect from the Pauli scheme, plus other terms that are typical of the particular, and firmly respected, approach. In the non-relativistic limit the terms that survive are, though, different from those derivable in the other scheme, since the scalar product with the electron spin which remains is not that of the magnetic field, but is that of the vector potential.

For the completeness of this search, the effects that an electric and a magnetic field would have on the purely intrinsic property of the electron which has been considered here, i.e. its spin, have also been computed. In the scheme that has been followed, the procedure is rather simple and starts form the changes of the spin currents, Equations (3)-(5), that are generated by the modifications of the psinons, Equations (9)-(12). This easily leads to the following results, denoted as usually by $\Delta_{A_{0}} S$ and $\Delta_{A} S$ :

$$
\begin{aligned}
& \Delta_{A_{0}} S=\frac{|e|}{m} \int \mathrm{d}^{3} x A_{0} \rho_{H} \\
& \Delta_{\boldsymbol{A}} \boldsymbol{S}=\frac{|e|}{m} \int \mathrm{d}^{3} x \boldsymbol{A} \wedge \boldsymbol{\rho}_{e}
\end{aligned}
$$

One sees that the same vector quantities $\rho_{H}, \rho_{e}$ that generate the energy changes in the Pauli schemes are producing in the minimal interaction approach the electric and magnetic effects, having changed their connections with the potentials, since $\rho_{H}$ interacts with $A_{0}$ and $\rho_{e}$ with $\boldsymbol{A}$. A final remark is thus that all the changes in the two relevant electron observables that I have considered, energy and spin, are produced by the combinations of the Pauli currents $\rho_{e}$ and $\rho_{H}$ with the electromagnetic potentials, with the addition of two typical terms generated by the chosen minimal interaction approach.

One final question is that of whether it might be possible to produce, at least qualitatively, an estimate of the two non-relativistic effects. With this purpose I have considered two values of the electric and magnetic fields that have been suggested to us by a dedicated medical equipe [3]. More precisely I have taken the values (for zero frequency fields)

$$
\begin{aligned}
& |\boldsymbol{E}|=130 \text { Volt } / \mathrm{m} \\
& |\boldsymbol{H}|=5 \times 10^{-5} \text { Tesla }
\end{aligned}
$$

In a super-simplified approach, some "average" values for $A_{0}$ and $\boldsymbol{A} \equiv\left\langle A_{0}\right\rangle,\langle\boldsymbol{A}\rangle$ have been obtained by assuming that the latter ones can be expressed by multiplying the fields intensity by a suitable length $=l \times 1$ meter assumed to be the same for $A_{0}$ and $\boldsymbol{A}$. Taking for the direction of $\boldsymbol{A}$ that of the $z$-axis, I obtain in the chosen units system

$$
\begin{aligned}
& \left\langle A_{0}\right\rangle \simeq l|\boldsymbol{E}| \times 100 \mathrm{~cm} \simeq l \times 4 \times 10^{2} \mathrm{eV} \\
& \langle\boldsymbol{A}\rangle \simeq l|\boldsymbol{H}| \times 100 \mathrm{~cm} \simeq l \times 5 \times 10^{2} \mathrm{eV}
\end{aligned}
$$

In this qualitative calculation, one sees that the intensity of the scalar and vector potentials are essentially the same. To derive the effect on the energy, one can retain in the defining integrals Equations (21), (22) the average potentials and obtain (with $\boldsymbol{A}$ in $\mathrm{Z}$ direction )

$$
\begin{aligned}
& \Delta_{A_{0}} \mathcal{H}_{N R L} \simeq|e|\left\langle A_{0}\right\rangle \int \mathrm{d}^{3} x \rho_{Q} \simeq 0.3 \times 10^{2} \mathrm{eV} \times l \times \int \mathrm{d}^{3} x \rho_{Q} \\
& \Delta_{\boldsymbol{A}} \mathcal{H}_{(N R L)} \simeq-4|e|\langle\boldsymbol{A}\rangle \int \mathrm{d}^{3} x s_{3} \simeq-1.7 \times 10^{2} \mathrm{eV} \times l \times \int \mathrm{d}^{3} x s_{3}
\end{aligned}
$$


A possible conclusion to be drawn from this very qualitative analysis is that the electric effect appears to be always positive since it is always $\rho_{Q(N R L)}>0$. The magnetic one depends on the values of $s_{3}$, which may generate a positive or negative $1 / 2$ integral. Assuming for $\int \mathrm{d}^{3} s_{3}$ a $\pm 1 / 2$ value and $\int \mathrm{d}^{3} x \rho_{Q}=1$ one sees that the magnetic effect for the considered field values is larger than the electric one. The final value depends on the choice of $l$, which might be assumed in practical case to be approximately one [4]. The possibly relevant observation is, in my opinion, that the magnetic effect strongly depends on the intrinsic spin of the considered electron or, alternatively, as already stressed, on the relative direction of the magnetic potential with respect to the electron spin. Changing this direction would change the (apparently dominant) magnetic effect on the considered electron. This conclusion might provide a useful suggestion for a suitable future medical experiment.

\section{Conclusions}

One clean conclusion that I draw from this analysis is that all the possible changes of the energy of an elementary electron state under the effect of general electric and magnetic fields only depend on two intrinsic property of the electron, i.e. its charge and its spin. I have shown that the considered process can be defined, as in my approach, a "fermion epigenetic" one, since all the visible effects on energy and spin are generated by changes of the four electron "psinons" which recall the analogous chain of effects that are generated in the human organic cells under the action of magnetic fields. A possible relevant conclusion that may be derived is that the size of magnetic and electric effects for realistic values of the fields is comparable and that, in particular, the magnetic effect depends on the space orientation of the magnetic potential $\boldsymbol{A}$, which is orthogonal to that of the magnetic field.

The possibility that these conclusions might have some relevance for the fascinating medical study of epigenetics is totally based on the belief that there might exist certain analogies between the evolution of the elementary matter components and the evolution of complex living organisms, as stated in a beautiful book written by A. Lima-de-Faria [5]:

"The evolution of living systems is a continuation of that of the physical world".

This possibility is at the moment being experimentally examined [3], and I am willing and ready to collaborate with the components of the group.

\section{Acknowledgements}

I am deeply indebted with my friend E. Spallucci for his precious suggestions and useful discussions.

\section{References}

[1] Burigana, F., Spallucci, E. and Verzegnassi, C. (2013) Journal of Modern Physics, 4, 1133-1138. http://dx.doi.org/10.4236/jmp.2013.48152

[2] Spallucci, E. and Verzegnassi, C. (2014) Journal of Modern Physics, 5, 99-102. http://dx.doi.org/10.4236/jmp.2014.53016

[3] P. Biava, Boffelli, private communication

[4] G. Giannini, private communication.

[5] Lima-de-Faria, A. (2003) Evoluzione senza selezione. Nova Scripta Edizioni, Genova. 
Scientific Research Publishing (SCIRP) is one of the largest Open Access journal publishers. It is currently publishing more than 200 open access, online, peer-reviewed journals covering a wide range of academic disciplines. SCIRP serves the worldwide academic communities and contributes to the progress and application of science with its publication.

Other selected journals from SCIRP are listed as below. Submit your manuscript to us via either submit@scirp.org or Online Submission Portal.
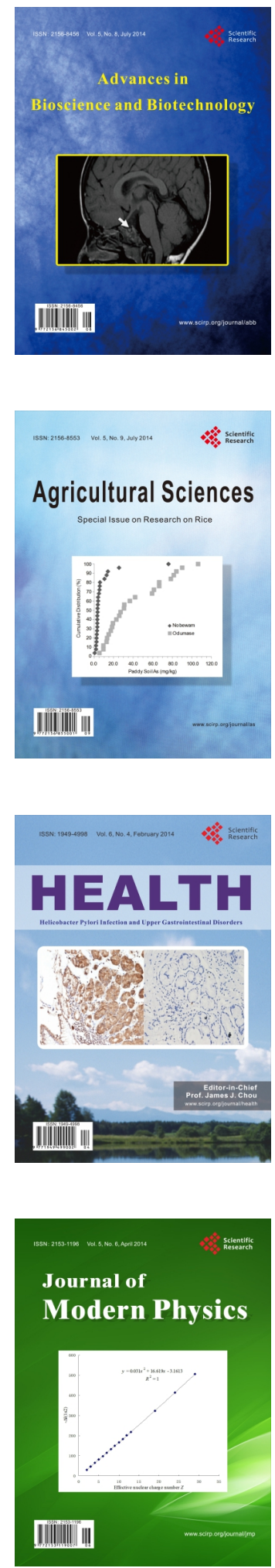
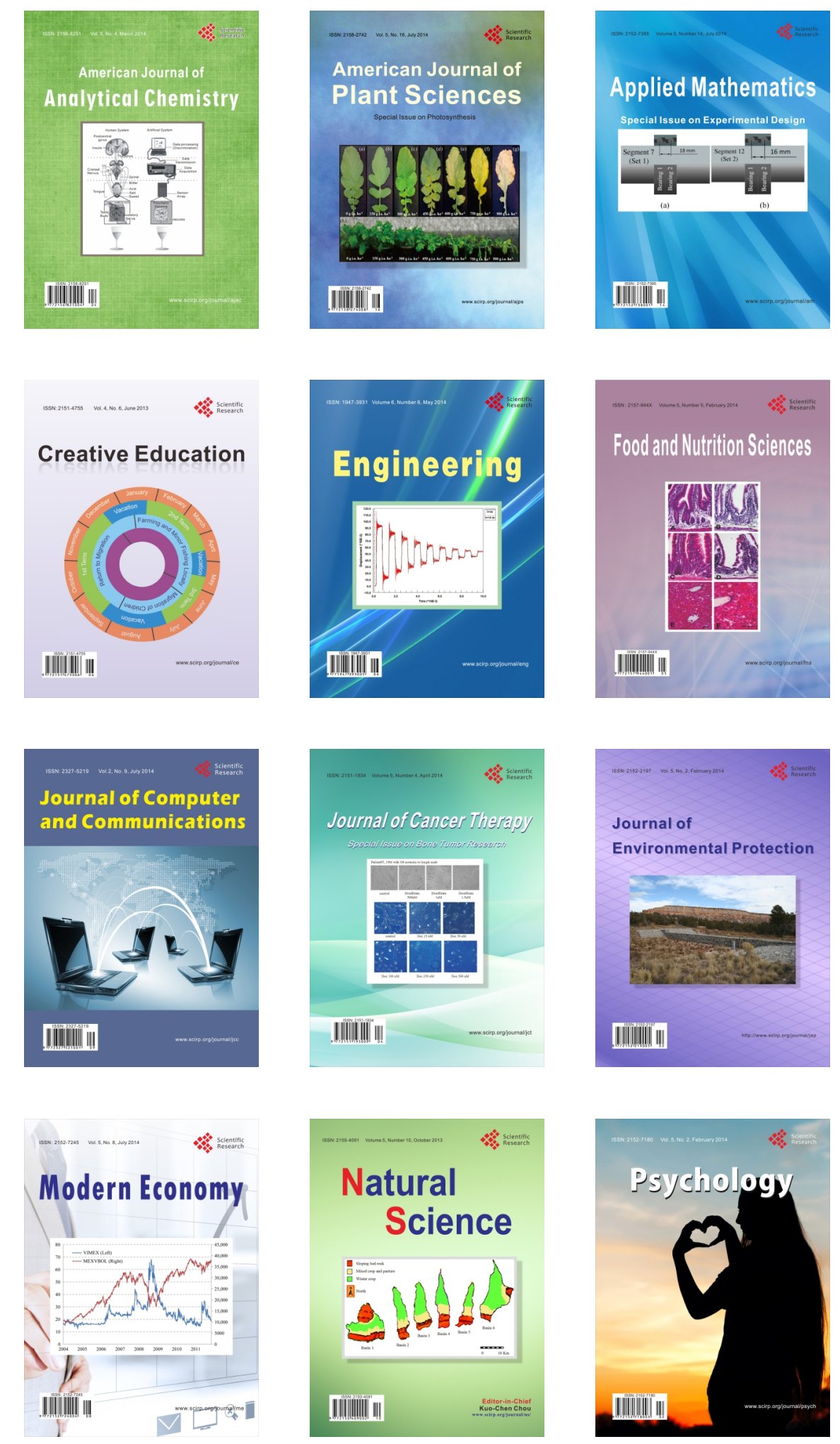